\title{
Vaccination and herd immunity to infectious diseases
}

\author{
Roy M. Anderson \& Robert M. May*
}

Department of Pure and Applied Biology, Imperial College, London University, London SW7 2BB, UK

* Biology Department, Princeton University, Princeton, New Jersey 08540, USA

\begin{abstract}
An understanding of the relationship between the transmission dynamics of infectious agents and herd immunity provides a template for the design of effective control programmes based on mass immunization. Mathematical models of the spread and persistence of infection provide important insights into the problem of how best to protect the community against disease.
\end{abstract}

MUCH attention is now focused on the development and community-wide use of vaccines for the control of infectious diseases. This interest is a consequence of many factors; these include the worldwide eradication of smallpox by vaccination in the late $1970 \mathrm{~s}^{1}$, the current success of child immunization programmes for the control of measles, mumps, pertussis and rubella in the United States ${ }^{2,3}$, the failure of frequent appeals by public health authorities in the United Kingdom to raise vaccination rates that are at present low by developed-country standards ${ }^{4-7}$, the Expanded Programme on Immunization (EPI) of the World Health Organisation (WHO) for the control of a variety of serious childhood viral and bacterial diseases in the developing world ${ }^{8-12}$, and the rapid advances in molecular biology and biotechnology which promise new vaccines for the future $^{13-16}$. The main thrust of research today is at the molecular level, where it is stimulated by the urgent need to develop vaccines for the major killing diseases of the Third World such as malaria ${ }^{17-19}$, and to combat new infections such as the current epidemic of AIDS (acquired immune deficiency syndrome), caused by the recently isolated human T-lymphotropic virus (HTLV-III) ${ }^{20,21}$

The development of a safe, effective and cheap vaccine, however, is only a first step (albeit an essential one) towards community-wide control. Epidemiological, economic and motivational issues are at least as important as technological ones. A vaccine for measles (a very cost-effective immunization ${ }^{12}$ ) has been available since the late 1960 s, yet the infection remains one of the world's major causes of child mortality ${ }^{8.22}$. Concomitant with research on vaccine development, there is a need for an improved understanding of how best to use vaccines to protect the community as well as the individual. The persistence of infectious disease within a population requires the density of susceptible individuals to exceed a critical value such that, on average, each primary case of infection generates at least one secondary case. It is therefore not necessary to vaccinate everyone within a community to eliminate infection; the level of herd immunity must simply be sufficient to reduce the susceptible fraction below the critical point. The central epidemiological questions are thus: what proportion of the population should be vaccinated to achieve elimination (in a local programme), eradication (in a global programme) or a defined level of control? How is this affected by demographic factors (for example, birth rate)? What is the best age at which to immunize? How does mass immunization affect the age distribution of susceptible individuals, particularly in those classes most at risk from serious disease, and how important is genetic and spatial heterogeneity in suceptibility to infection (or response to immunization) to the creation of effective herd immunity ${ }^{23-26}$ ?

To answer these questions, we need to understand the interaction between the transmission dynamics of the disease agent and the level of naturally acquired (or artificially created) immunity to infection. This relationship is complex and depends on such factors as the precise course of infection within an individual, the demography of the host population, the duration of acquired immunity and maternally derived protection, agerelated changes in the degree and intimacy of contacts among people, and the prevailing levels of genetic, spatial and behavioural heterogeneity in susceptibility/resistance to infection. Intuition built on many years of practical epidemiological experience often fails to predict the outcome of a particular vaccination programme. Mathematical models which clearly and accurately define the details of the association between disease agent and host (at the individual and population levels) can help to overcome such difficulties. This article reviews recent theoretical and empirical work on the transmission dynamics of infectious disease. Emphasis is placed on the relevance of such research to the design of disease control programmes based on vaccination.

\section{General theory}

Models for the transmission dynamics of directly transmitted viral and bacterial infections are typically compartmental in structure $e^{23-35}$. They represent changes, with respect to age, $a$, and time, $t$, in the populations of infants protected by maternally derived antibodies $(M(a, t))$, susceptible individuals $(X(a, t))$, infected individuals who are not yet infectious $(H(a, t))$, infectious people $(Y(a, t))$, and people who have recovered and are immune from subsequent infection $(Z,(a, t))$. The total population is $N(a, t)=M(a, t)+X(a, t)+H(a, t)+Y(a, t)+Z(a, t)$. The deterministic equations describing the flows from one class to another are of the general form ${ }^{24,25,27}$

$$
\begin{aligned}
& \frac{\partial M(a, t)}{\partial t}+\frac{\partial M(a, t)}{\partial a}=-[\mu(a)+d] M(a, t) \\
& \frac{\partial X(a, t)}{\partial t}+\frac{\partial X(a, t)}{\partial a}=\mathrm{d} M(a, t) \\
& -[v(a, t)+\lambda(a, t)+\mu(a)] X(a, t) \\
& \frac{\partial H(a, t)}{\partial t}+\frac{\partial H(a, t)}{\partial a}=\lambda(a, t) X(a, t) \\
& \frac{\partial Y(a, t)}{\partial t}+\frac{\partial Y(a, t)}{\partial a}=\sigma H(a, t)-[\mu(a)+\gamma] Y(a, t) \\
& \frac{\partial Z(a, t)}{\partial t}+\frac{\partial Z(a, t)}{\partial a}=\gamma Y(a, t)+v(a, t) X(a, t) \\
& \frac{\partial N(a, t)}{\partial t}+\frac{\partial N(a, t)}{\partial a}=-\mu(a) N(a, t)
\end{aligned}
$$

Here $\mu(a)$ denotes age-related human mortality, $1 / d$ is the average duration of protection provided by maternal antibodies, $1 / \sigma$ and $1 / \gamma$ are the average incubation and infectious periods respectively, and $v(a, t)$ records the age- and time-specific vaccination schedule. Mortality explicitly associated with the 
Fig. 1 a Time series analysis of the case resports for measles in England and Wales over the pre-vaccination period 1948-68. The top graph denotes the recorded cases and the bottom graph the serial correlogram ${ }^{51}$. Note the significant yearly (seasonal) and 2-yearly peaks in incidence (the horizontal axis in the bottom graph denotes weeks). $b$, Model predictions of the equilibrium (the state to which the system settles in the long term) ratio of the number of cases of mumps in the age range $14-50 \mathrm{yr}$ (the age range in which males are at risk to serious disease resulting from infection) under the impact of a vaccination programme in which $p$ of each yearly cohort are vaccinated at age $2 \mathrm{yr}$, divided by the number of cases in the same age range before vaccination. If the ratio is above unity, the control programme has a deterimental effect and vice versa. The average age at infection before control was assumed to be $6.5 \mathrm{yr}$ (appropriate for the United Kingdom) and the calculations were based on a model defined in ref. 24. The straight line denotes the ratio of unity. $c$ Age-specific incidence of reported measles encephalitis cases (defined per 1,000 cases of measles) in the United States in 1973$79^{116}, d$, Similar to $b$ but representing the ratio of cases after vaccination divided by cases before vaccination for rubella in the United Kingdom in the age range $16-40 \mathrm{yr}$. The graph denotes model predictions (equilibrium values) for a two-stage vaccination policy involving vaccination of only girls at age $12 \mathrm{yr}$ (the UK policy) and vaccination of boys and girls at age $2 \mathrm{yr}$ (the US policy) ${ }^{25,67}$. Note that the addition of a second-stage US policy over the current UK policy is only of substantial benefit if moderate to high levels of vaccination coverage are achieved at 2 yr of age. The average age at infection before control was assumed to be 9-10 yr of age $\mathrm{y}^{25,67}$.
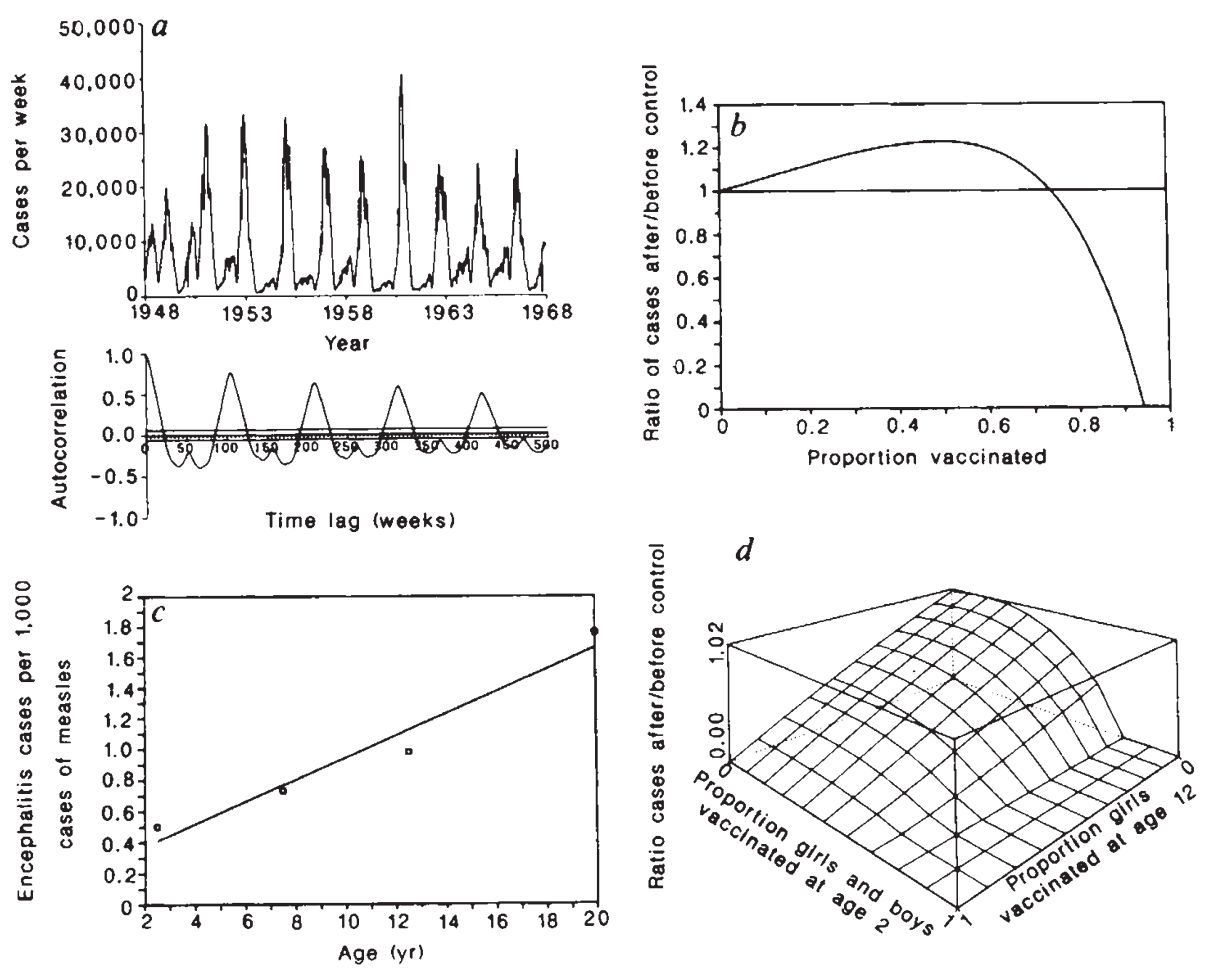

infection has been ignored. The term $\lambda(a, t)$ denotes the 'force' or rate of infection, which under the 'mass action' assumption adopts the form ${ }^{24-30} \lambda(a, t)=\int_{0}^{\infty} \beta\left(a, a^{\prime}\right) Y\left(a^{\prime}, t\right) \mathrm{d} a^{\prime}$. Here $\beta\left(a, a^{\prime}\right)$ represents the transmission coefficient for infected individuals in age class $a^{\prime}$ who come into contact with susceptible individuals in age class $a$ (refs 25,30 ). The boundary conditions of equations (1) depend on the demography of the community. The structure of the model may be modified to mimic the transmission of a wide variety of infections ${ }^{31-34}$, including those with complex life cycles (for example, malaria or yellow fever ${ }^{35-37}$ ) and those involving carriers (such as tuberculosis and hepatitis $\mathrm{B}^{38}$ ) or macroparasites (such as helminths) where note must be taken of the distribution of parasite numbers within the human community ${ }^{39,40}$.

Insights into applied problems of disease control or into general ecological and epidemiological questions can be obtained through analytical or numerical studies of equations (1).

\section{Cycles in incidence}

For most common viral and bacterial infections of childhood, the model predicts large-amplitude, slowly damping oscillations in disease incidence ${ }^{23-30}$. Several factors can tend to perpetuate these oscillations indefinitely; these are stochastic effects ${ }^{41-44}$, age-dependent transmission rates ${ }^{25}$, incubation periods of a discrete nature ${ }^{45,46}$ and seasonality in contact ${ }^{27,47-50}$. The average period of oscillations, $T$, is approximately given $\mathrm{as}^{23}: T \simeq$ $2 \pi(A K)^{1 / 2}$, where $A$ is the average age at infection and $K$ is the generation time of the infection $(K=1 / \sigma+1 / \gamma)$.

The observed periods of such cycles (which agree well with prediction) can be determined accurately by time-series techniques (Table $1 a$, Fig. $1 a^{51}$ ); the longer-term cycles are usually compounded with short-term seasonal effects.

\section{Vaccination and herd immunity}

The basic reproductive rate or transmission potential of an infection, $R_{0}$, is the average number of secondary cases produced by one primary case in a wholly susceptible population ${ }^{33,34}$. Clearly, an infection cannot be maintained unless $R_{0}$ exceeds unity. Within communities in which the infection is endemic, $R_{0}$ is inversely correlated with $A$, the average age at infection ${ }^{23-37}$ $\left(R_{0} \simeq B /(A-D)\right)$. This relation holds both for developed countries (growth rates essentially zero) and for developing countries (with their growing populations) ${ }^{52}$. Here $B$ is the reciprocal of the finite birth rate (usually expressed as births $\left(\mathrm{yr}^{-1}\right)$ per 1,000 of population), and $D$ is the duration of maternally derived immunity. The value of $R_{0}$ within a community is critically dependent on social and behavioural factors (the contact component of $\beta$ which influences $\lambda$ and hence $A$ ), on the biology of the disease agent and host (the infectiousness and susceptibility components of $\beta$ ) and on the demography of the community (value of $B)^{25}$. The marked difference between developed and developing countries in the average ages at which children typically acquire infections such as measles in urban communities is a consequence of variations in social, behavioural and demographic factors (Table $1 b$ ).

To eradicate an infection by mass immunization it is necessary to reduce the value of $R_{0}$ below unity ${ }^{23-25,53}$. This can be achieved by vaccinating a proportion, $p$, of each cohort of the population at age $V$ (where $V>D$; vaccination during the period of maternally derived protection usually fails to protect the child adequately from subsequent infection ${ }^{54,55}$ ) provided $^{23,25} p>$ $[1-A / B] /[1-V / B]$. The infection can never be eradicated if $V>A$ (that is, $p>1)$. The value of $p$ is minimized by vaccinating at as young an age as possible, given the constraint of $V>D$. These conclusions are derived under the assumption that the rate of transmission within the community is fairly homogeneous. In practice, this assumption is often violated ${ }^{25,30}$, but simple theory provides a useful guide, usually setting an upper limit to the levels of vaccination required to eliminate many common childhood infections ${ }^{25}$. It forms the basis of estimates suggesting that $92-96 \%$ of children must be vaccinated to eliminate measles and pertussis, $84-88 \%$ to eliminate rubella and $88-92 \%$ to eliminate mumps in Western Europe and the United States; these estimates assume that vaccination takes place just after the wane of maternal antibody protection ${ }^{23-25}$. Recent experience in the United States suggests that high levels of cohort immunization are required (as indicated by theory) 
Table 1 Infections reported throughout the world

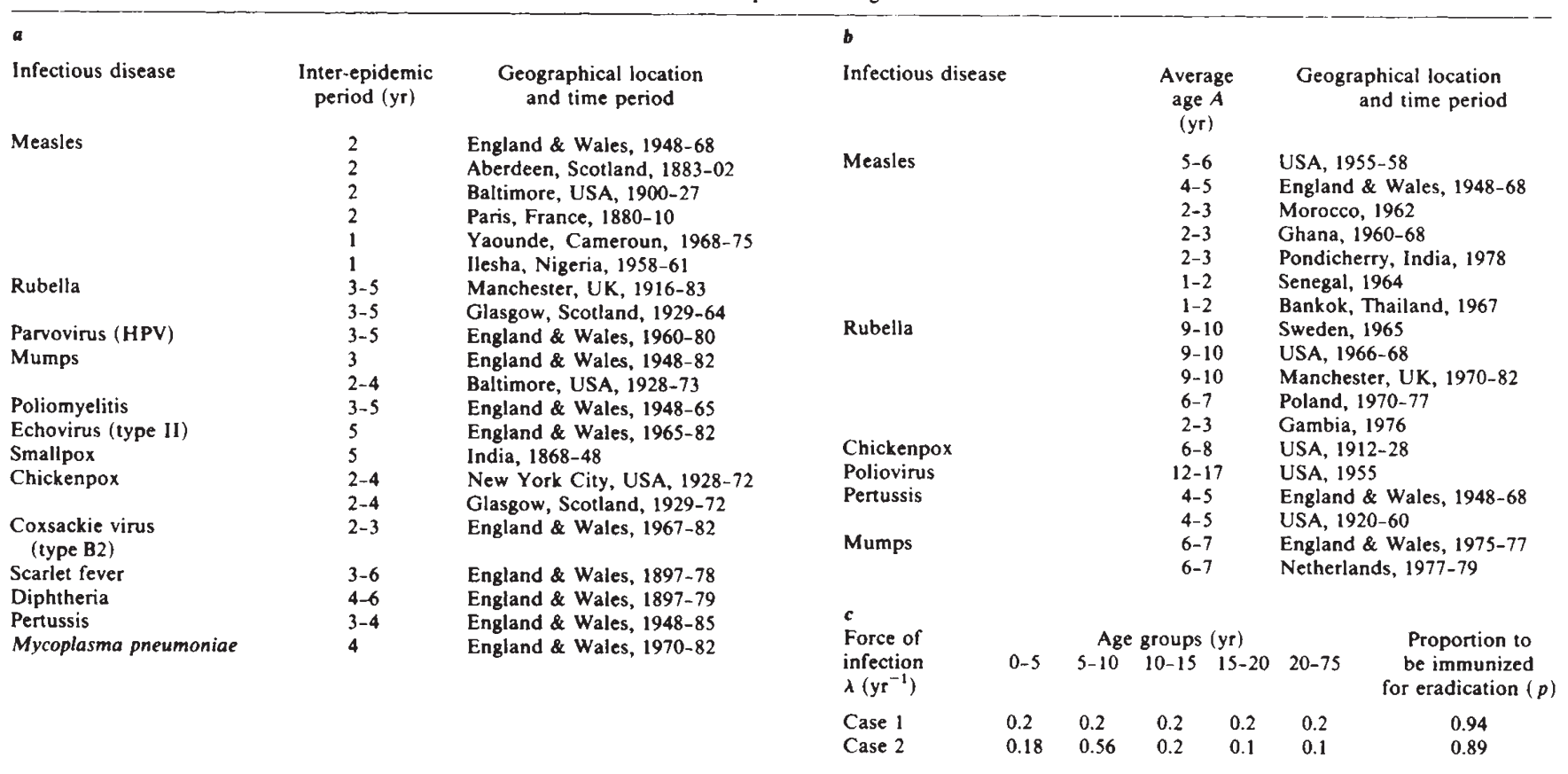

$a$, The inter-epidemic period, $T$, for various viral and bacterial infections ${ }^{23-25,51,99,115}, b$, Average age at infection (yr), $A$, for different diseases in different localities ${ }^{23,24,34,99,116}$. $c$, The impact of age-related changes in the force of infection, $\lambda\left(\mathrm{yr}^{-1}\right)$, on the critical proportion of the population $p$, to be vaccinated to eradicate measles in England and Wales ${ }^{23-26}$ (case 1, constant; case 2 age dependent).

to substantially reduce the incidences of common childhood infections.

The vaccination of cohorts must be continued for many decades before the full impact of a programme is manifest; long-term suppression or interruption of transmission requires continual cohort vaccination as long as the infection remains endemic in other communities or countries. The only infection to be eradicated worldwide is smallpox. This success (announced by WHO in 1977) generated some optimism concerning the feasibility of eradicating other major infections, such as measles and pertussis ${ }^{8,9,56-58}$. Such optimism, however, may be misplaced, given the low communicability of smallpox (low $R_{0}$ and high average age at infection, $A$, before widespread immunization) in comparison with infections such as measles, the ease with which smallpox infection can be diagnosed, the practical simplicity of inoculation procedures and the stability of the smallpox vaccine under primitive storage conditions ${ }^{1,59,60}$.

Successful immunization programmes may themselves create health problems, since vaccination almost invariably carries some risk (usually extremely small) to the individual. At the start of a mass-immunization programme, the probability of serious disease arising from vaccination is usually orders of magnitude smaller than the risk of serious disease arising from natural infection. As the point of eradication is approached, the relative magnitude of these two probabilities must inevitably be reversed. The optimum strategy for the individual (not to be vaccinated) therefore becomes at odds with the needs of society (to maintain herd immunity). This issue-which was central to the decline in uptake of whooping cough vaccine in the United Kingdom during the mid- to late-1970s-can be overcome by legislation to enforce vaccination (as in the United States), but its final resolution is only achieved by global eradication of the disease agent (so that routine vaccination can cease) ${ }^{61}$.

Mass immunization at levels below that required for elimination acts to reduce the number of cases of infection (and hence the force of infection, $\lambda$ ). However, both theory ${ }^{23-26}$ and observation $^{62,63}$ suggest that it has little, if any, impact on the total number of susceptible individuals. If the infection is endemic, its effective reproductive rate is unity-each case produces, on average, one other case-and the fraction susceptible remains roughly constant $\left(\sim 1 / R_{0}\right)$, independently of whether suscepti- bility is lost by vaccination or by natural infection. Reduction of transmission via vaccination, however, usually increases the inter-epidemic cycle period and the average age at infection $^{23-26,51}$. This latter manifestation can be worrying if the risk of serious disease resulting from infection increases with age (Fig. 1c). Whether this issue is important depends on the quantitative details of such factors as how risk changes with age, the average age at which the vaccine is administered, and how the force of infection changes with age before widespread immunization $^{25,26}$ (Fig. 3a). Rubella and mumps are of particular concern because of the risk of congenital rubella syndrome (CRS) in infants born to mothers who contracted rubella in their first trimester of pregnancy ${ }^{64,65}$ and the occurrence of orchitis and the associated risk of sterility in post-pubertal males following mumps infection ${ }^{66}$ (Fig. 1b,c). The problems surrounding mass vaccination against rubella (to control CRS) have resulted in the adoption of different vaccination programmes in different countries. In the United Kingdom, girls only are vaccinated at an average age of around $12 \mathrm{yr}$, so as to allow rubella virus to circulate and create naturally acquired immunity in the early years. By contrast, in the United States both boys and girls are vaccinated at around $2 \mathrm{yr}$ of age, with the aim of eliminating rubella. Theory suggests that the US policy is best if very high levels of vaccination $(80-85 \%$ of each yearly cohort) can be achieved at a young age, while the UK policy is better if this cannot be guaranteed ${ }^{24,67}$. A mixed US and UK policy is predicted to be of additional benefit over the UK single-stage policy if moderate to high levels of vaccine uptake among boys and girls can be achieved at a young age $(>60 \%)^{67}$ (Fig. 1d).

\section{Epidemiological data}

The average age at infection, $\boldsymbol{A}$, is central to most estimates of the intrinsic transmission potential of an infection, to the calculation of the desired level of vaccination coverage for elimination, and to theoretical assessment of how the risk of serious disease caused by infection may change with age under the impact of mass vaccination. For infections which are thought to induce life-long immunity against disease (for example, measles, mumps, rubella and pertussis), the value of $A$ can be estimated (see Table $1 b$ ) either from age-stratified case notification records or, more accurately, from serological profiles (Fig. 2). 

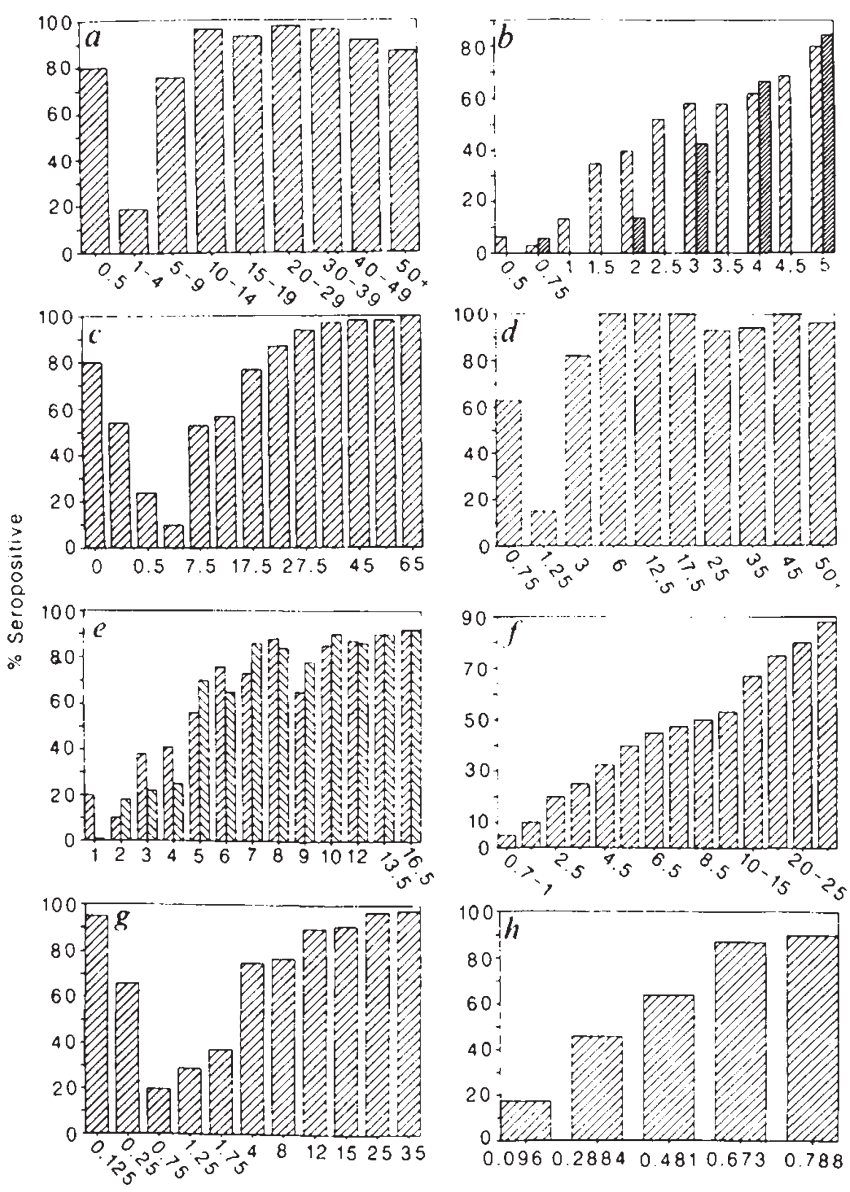

Fig. 2 Age-stratified (horizontal) serological profiles of the percentages of people in different age groups with antibodies to various infectious disease agents in developed and developing countries. $a$, Measles in the United States in 1955-58 (New Haven, Connecticut) ${ }^{i 17}$. $b$, Measles in India ${ }^{118}$ (left-hand histograms) and Nigeria ${ }^{119}$ (right-hand histograms). Note the rapidity with which the proportion seropositive rises with age when compared with a. c, Rubella in England and Wales ${ }^{24}$ in the 1960s and 1970s.d, Rubella in the Gambia in the late $1970 \mathrm{~s}^{120}$. Again note the rapidity of the rise in seropositivity with age when compared with c. $e$, Mumps in England and Wales (left-hand histograms) and the Netherlands (right-hand histograms) $)^{121,122} . f$, Polio in the United States in $1955^{34} . g$, Hepatitis B (HBV) in Senegal ${ }^{123}$. The vertical axis denotes the percentage with HBV markers. $h$, Malaria (Plasmodium falciparam) in Nigeria ${ }^{115}$. The vertical axis denotes the cumulative percentage of children who have experienced infection. Note that in $a-h$ the scales of the $x$-axes are not always identical and in certain cases record unequal age class divisions (a nonlinear scale).

The interpretation of the data, however, is beset with many problems, such as age-specific biases in notification records ${ }^{68,69}$, and a reduced ability to detect antibodies in adults who experienced infection in early childhood ${ }^{70}$.

\section{Heterogeneity in transmission}

Conventional epidemiological theory is founded on the 'mass action' principle of transmission (see equations (1)), in which infection is assumed to spread via contacts within a homogeneously mixing community ${ }^{71}$. Recent research has begun to take account of the complications induced by heterogeneity in transmission arising from age-related, genetic, spatial or behavioural factors $25,30,72-77$.

Age-dependent factors. Analyses of case notification records and serological profiles suggest that, for many common infections (measles, rubella and pertussis), the per capita rate of infection $(\lambda(a, t))$ depends on the ages of susceptible individuals, changing from a low level in the $0-5$-yr age classes, via a high level in the 5-15-yr age classes, back to a low level in the adult age classes (Fig. $3 a)^{25}$. This is of interest both because it reflects behavioural attributes of human communities and because of its impact on the level of vaccine-induced herd immunity required to eliminate an infection ${ }^{25,67,75}$. The high values of $\lambda$ observed in the 5-15-yr-old classes (Fig. $3 a$ ) are thought to arise as a consequence of frequent and intimate contacts within school environments.

Analyses of the significance of age-related changes in contact with infection are crude at present, due to the absence of detailed information on "who acquires infection from whom, 25 . Models which incorporate high degrees of contact within school-age classes (and low contact in the very young and in adult age groups), however, yield predictions of temporal changes in disease incidence and of age-related changes in serology in vaccinated versus unvaccinated communities which closely parallel observed trends ${ }^{25}$ (Figs $3 c, d, 4 a-c$ ). Recent numerical studies suggest that predictions based on the assumption of homogenous mixing tend to overestimate the critical level of vaccination coverage for elimination by roughly $5 \%$ for infections such as measles and pertussis in the United Kingdom and United States (Table $1 c)^{25,30}$. For these infections, analyses based on apparent age-related changes in contact with infection (Fig. $3 a$ ) yield estimates of approximately $90 \%$ coverage at 1-2 yr of age $\mathrm{e}^{25}$. These predictions, however, must be accepted with caution at present, because the observed low rates of infection in late teenage and adult age groups may be artefacts arising from biases in case reporting, from the insensitivity of serodiagnostic tests, or from genetic variability in susceptibility to infection ${ }^{78}$. These caveats are underlined by the fact that no such marked age-related changes in the per capita rate of infection are found in epidemics within virgin populations on islands (largely susceptible to infection before the epidemic ${ }^{79-81}$ ) (Fig. $3 b$ ). At present it seems prudent to accept the higher vaccine coverage levels estimated by homogeneous mixing models ${ }^{25}$.

Genetic factors. Genetic factors are important in determining host susceptibility and resistance to infectious agents ${ }^{78}$. Genetically based variability in the level and duration of antibody production following infection would seriously complicate the interpretation of age-serological profiles ${ }^{82}$. Associations between HLA type and antibody titres following vaccination against rubella and measles provide evidence for the existence of such complications ${ }^{83,84}$. Little is understood, at present, about the importance of such factors in the design of vaccination programmes. In the future, however, community-based diagnostic programmes aimed at the identification of immunodeficiency could make it possible to target vaccination, for certain lesscommon but serious viral and bacterial diseases, to those children genetically predisposed to infection and severe morbidity ${ }^{85}$. Spatial factors. Inhomogeneity in the spatial distribution of hosts, with some people living in dense aggregate and others living in isolated or small groups, can lead to heterogeneity in transmission rates. This, in turn, can result in the transmission potential of an infection $\left(\boldsymbol{R}_{0}\right)$ being greater on average than suggested by estimation procedures which assume spatial homogeneity ${ }^{86,87}$. Under these circumstances, the optimal solution appears to involve 'targeting' vaccination coverage in relation to group size, with dense groups receiving the highest rates of vaccination ${ }^{74}$. The optimal programme is defined as that minimizing the total, community-wide number of immunizations needed for elimination or a defined level of control. This strategy reduces the overall proportion that must be vaccinated to achieve elimination, compared with that estimated on the assumption of spatial homogeneity. This conclusion has practical significance for the control of infections such as measles and pertussis in some developing countries, where rural-urban differences in population density tend to be much more marked than in developed countries. It is probable that in many regions of Africa and Asia, diseases such as measles cannot persist endemically in rural areas without frequent movement of people between rural and urban regions $\left(R_{0}<1\right.$ in rural areas). Under these circumstances, disease control might be achieved in both types of region by high levels of mass immunization in the urban centres alone. 
Fig. 3 a, Age-specific change in the rate, or force, of measles infection $(\lambda)$ in England and Wales before mass vaccination ${ }^{25}$. $b$, Percentages seropositive for rubella antibodies in various age classes on St Paul's Island, Alaska, before (left-hand histograms) and after (right-hand historgrams) an epidemic of rubella in $1963^{79-81}$. The population ( -400 people) had not experienced rubella infection (before 1963) for $22 \mathrm{yr}$. Note that in the age range $0-19 \mathrm{yr}$, the rate of infection of susceptible individuals was similar in each age class. $c$, Comparison of model predictions (equations (1)) of the age-serological profile for measles antibodies in England and Wales with observed patterns. The observed data (squares̀) were collected in the period 1979-82. Model predictions were based on an average age of infection of $4.5 \mathrm{yr}$ before control and the agespecific vaccination rates in the United Kingdom over the period $1968-82^{25}$. $d$, Comparison of model predictions of the age-serological profile for rubella antibodies in females in England and Wales with the observed pattern (crosses) in $1984^{67}$. Predictions were based on an average age of infection of $10 \mathrm{yr}$ before control and the age-specific vaccination rates in the United Kingdom (vaccination of teenage girls between the ages of 10 and $15 \mathrm{yr}$ ) over the period 1970-84.
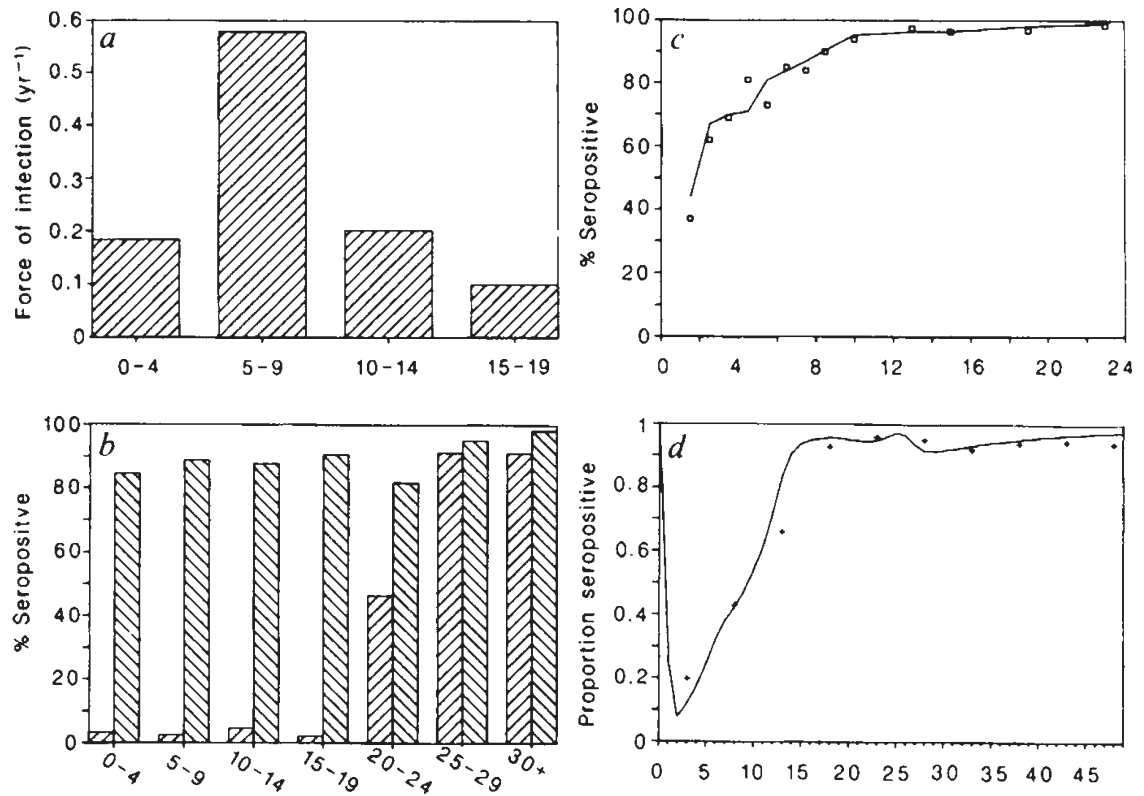

Age class (yr)

Age (yr)
Behavioural factors. These are of great importance as determinants of disease transmission and provide a source of significant heterogeneity. The sexually transmitted infections provide a good example because the probability distribution of sexual promiscuity (defined as the number of different sexual partners per unit of time) is highly skewed. It seems very likely that the highly sexually active individuals $\left(R_{0} \gg 1\right)$ in the tail of the distribution are responsible for maintaining transmission within the community as a whole ${ }^{88-90}$. Drug treatment and surveillance focused on these 'superspreaders' is therefore highly beneficial, given that $R_{0}$ may be below unity for the remaining population. If vaccines are developed for HTLV-III, selective immunization of those most at risk ${ }^{91-93}$ (haemophiliacs, intravenous drug abusers, persons of Central African or Haitian origin and homosexual males) and those predisposed to serious clinical infection, may be a practical option for community-wide control.

Behavioural factors are also important in helping to cause seasonal fluctuations in the incidence of many common viral and bacterial infections ${ }^{49,75,94}$. The timings of school terms and vacation periods has a marked influence on temporal patterns of measles and pertussis cases in the United Kingdom and West Germany, for example.

\section{Developing countries}

Many infections that in developed countries are primarily a source of morbidity are major cause of mortality in the developing world (mainly as a consequence of malnutrition, although genetic components may also be involved) $)^{9,22,95}$. Measles, for example, is estimated to cause more than one million child deaths annually ${ }^{9,22}$. Although there is much current interest in worldwide immunization programmes for the control of such infections, the problems of creating sufficient levels of herd immunity for elimination should not be underestimated, given the logistic difficulty of vaccine storage and delivery in poor countries. Even if such practical issues are resolved by effective cold-chains, the development of heat-stable vaccines, and aerosol delivery techniques (which are more effective in the immunization of infants with maternal antibodies $)^{96-98}$, the feasibility of blocking transmission by mass vaccination in areas where the average age of infection is often as low as 1-2 yr of age is questionable (Table $1 b$ ).

Consider a large urban centre in India or Africa, where the annual birth rate is around 40 per 1,000 population and the average age of measles infection is $2 \mathrm{yr}$ (Fig. 2). Vaccination must take place after the maternally derived protection has waned ( 6 months) and before the age of 2 yr to achieve elimination. Calculations suggest an $R_{0}$ value of around 17 , which is similar to that for measles in England and Wales (with a birth rate of 12 per 1,000 and an average age of infection around 4.5-5 yr). Simple theory suggests that something like $96 \%$ of each yearly cohort of children would have to be effectively immunized by age 1 yr $(V=1)$ to attain elimination. Coverage could be lower in rural areas but, as suggested in the section on spatial heterogeneity, an optimal control programme would have to focus on the urban centres. These estimates question the feasibility of measles elimination in many developing countries at this time and argue that, as a first priority, worldwide programmes should concentrate on controlling morbidity and mortality among those most at risk ${ }^{52.99}$. Moderate to low levels of vaccination coverage, however, are beneficial; they raise the average age at infection and thereby lengthen the age 'window' in which vaccine can be administered ${ }^{99}$, and reduce morbidity and mortality (young infants appear to be more at risk than older children).

\section{New vaccines}

The coming decade will see the development and use of a number of new vaccines, along with the expansion of community-based programmes of mass immunization using the currently available vaccines. The development of safe, effective and cheap vaccines is clearly the first priority, but, once these objectives are achieved, the problem of community-wide control requires careful attention. In particular, age-specific serological profiles of the community need to be obtained both before and during any programme of mass vaccination.

An example is provided by the recent proposal to initiate a mass-vaccination campaign for the control of hepatitis $B$ virus (HBV) in Taiwan ${ }^{14}$ by a serum-derived vaccine (a genetically engineered vaccine is also available $\mathrm{e}^{100,101}$; Fig. 2). The epidemiology of HBV is made complicated by chronic infection in immunodeficient individuals who become carriers of the virus (with a high associated risk of inflammatory disease and malignancy) $)^{102}$, and who constitute an important reservoir of infection. The desirability of mass immunization is clear when viewed as a method for reducing the incidence of carriers (the risk of becoming a carrier appears to be much greater in children than in adults ${ }^{103}$, but less clear given the observation that serious symptoms of disease are more likley to occur in adults than in children. Serological surveys in high-risk regions (South-East Asia and Africa) indicate that $\mathrm{HBV}$ typically has a low $\boldsymbol{R}_{0}$ within the population as a whole (when compared with measles). 
Moderate levels of vaccination coverage should therefore have a significant impact on disease incidence $e^{23,102,104}$ (Fig. 1d). The calculation of $R_{0}$ is made complicated by the presence of carriers $\left(R_{0}=R_{01} f+R_{02}(1-f)\right.$, where $f$ is the fraction of carriers and $(1-f)$ the fraction of non-carriers) and a component of perinatal (=vertical) transmission ${ }^{42}$. The maintenance of transmission $\left(R_{0}>1\right)$ is probably due to the carriers alone $\left(R_{01} \gg 1, R_{02}<1\right)$ given that they are often a significant fraction of the community (with $f$ values ranging from $0.1 \%$ to $2 \%$ in the United States and most European countries, to as high as $50 \%$ in certain Pacific islands (determined in part by the ethnic composition of a community $\left.{ }^{102}\right)$ ).

Vaccines for viral infections such as cytomegalovirus, rhinovirus, adenovirus, rotavirus, respiratory syncytial virus and parainfluenza virus may provide sufficient protection to the individual to be considered for community-wide use in the future, provided their public health significance is of sufficient importance relative to other priorities in health care. The existence of many distinct antigenic types within certain groups of these viruses (for example, adenovirus and rhinovirus), however, present problems for vaccine development.

Recent work has raised the hope that vaccines may be developed for the major parasitic infections such as malaria (Plasmodium falciparum) and certain helminth species ${ }^{105}$. The problems, however, are formidable because of the antigenic complexity of protozoa and helminths (in comparison with viruses and bacteria $)^{106}$, sequential changes in surface antigens during development within the host (for example, the filarial worms) ${ }^{107}$, antigenic variation (whether due to the parasite's ability to express many variable antigen types, as in the case of the African trypansomes ${ }^{108,109}$, or as a consequence of population genetic (=strain) variability, as in the case of the malaria parasites ${ }^{110}$, and the failure of man naturally to develop fully protective immunity under conditions of repeated exposure to infection ${ }^{111}$. Vaccines must be more effective in stimulating the host's immunological defences than the parasite itself if they are to overcome the evasion mechanisms that certain parasitic species have evolved ${ }^{112}$. Combined with these problems is the suspicion that heterogeneity in immunological responsiveness is an important determinant of the observed aggregation in the distribution of parasite numbers per person (such that $15 \%$ of the hosts will often harbour $90 \%$ or more of the parasites $)^{39,40,113,114}$. Predisposition to heavy or repeated parasitic infection is often a consequence of behavioural or social factors but laboratory studies increasingly point to the importance of genetic and nutritional components ${ }^{112}$. An effective vaccine must therefore be able to protect both immunocompetent and immunodeficient individuals, the latter being those most likely to suffer serious disease and to maintain parasite transmission.

The ideal malaria vaccine will probably contain antigens from different malaria species, strains and developmental stages (sporozoite, asexual and gamete) so as to reduce mortality and morbidity, and to block transmission ${ }^{18,19}$. But once the technical problems in vaccine development have been overcome, the issues of community control must take priority. The creation of a level of herd immunity high enough to eliminate malaria appears impractical in endemic regions where the average age at first infection is typically around 3-6 months of age $\left(R_{0}=\right.$ 50-100, which implies $99 \%$ coverage of the community before the age of 3 months with a vaccine which gives lifelong protection) (Fig. 1e). Low to moderate levels of immunization, however, will greatly help to control mortality and morbidity, which is of greatest significance in the $0-5$-yr-old age classes. Care must, however, be exercised to prevent an associated build-up of susceptible individuals in teenage and adult age classes; at present, such individuals have acquired a degree of immunity to infection from repeated exposure in childhood. Once the properties of a potential vaccine are more clearly defined, mathematical studies can help to quantify such risks. In the case of helminth infections, vaccines will probably only provide protection of short duration against re-infection, and
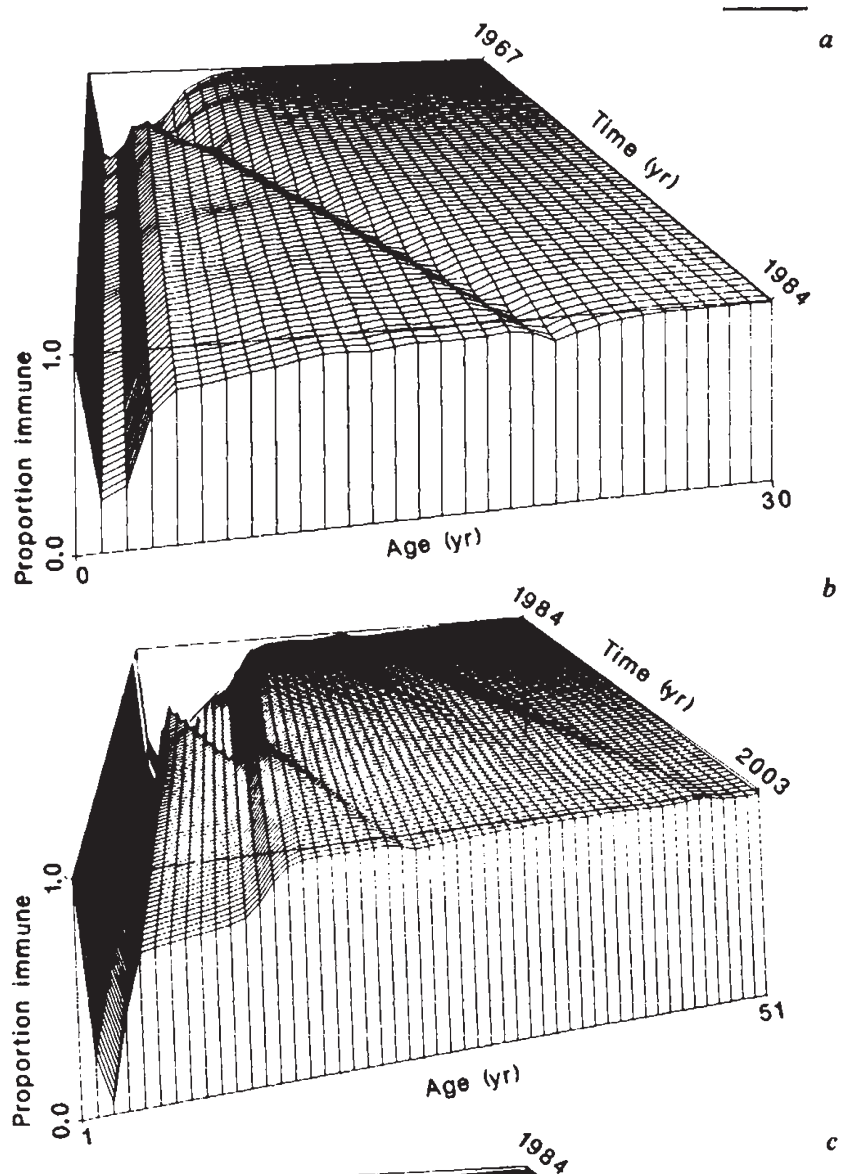

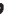

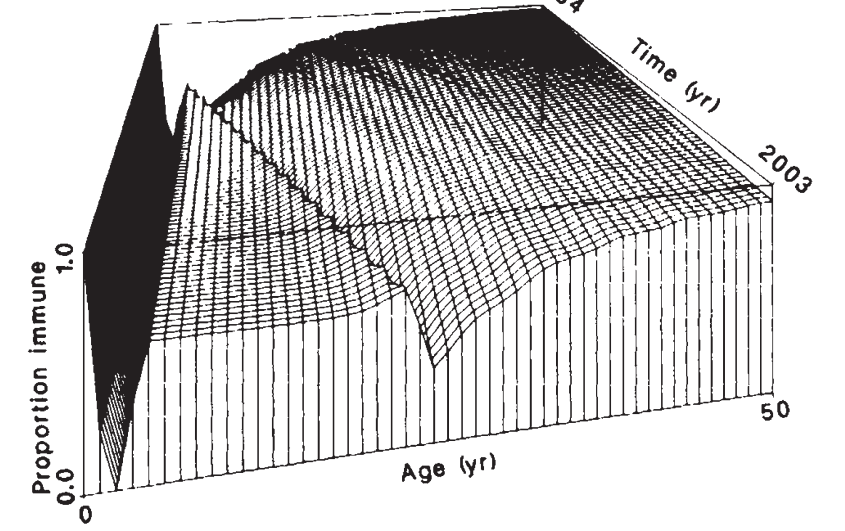

Fig. 4 a, Model predictions (equations (1)) of the impact of mass vaccination on the age-serological profiles for measles antibodies (the proportion in each age class who are immune either as a result of maternally derived protection (in the infants), recovery from natural infection, or vaccination) in England and Wales ${ }^{25}$. Mass vaccination was introduced in year 1 (year 0 denotes the pre-vaccination profile, based on the age-specific forces of infection defined in Fig. $3 a$ ) and maintained for $16 \mathrm{yr}$ with the age-specific vaccination rates recorded in England and Wales (mean age at vaccination $\sim 2.2 \mathrm{yr} ;-50-60 \%$ of each yearly cohort was immunized by age $5 \mathrm{yr}$ ). The age axis denotes the intervals $0,0.5,1.0,2.5, \ldots$ etc. yr. Note how mass vaccination (in the age range $1-3 \mathrm{yr}$ ) acts to decrease the proportion seropositive in the older age classes (by reducing the overall force of infection within the community below the level in the pre-vaccination community). The valley of seronegativity running diagonally across the surface denotes the ageing of the last cohorts of young children to be excluded from mass immunization (see ref. 25 for further details). $b, c$, Similar to $a$ but denoting model predictions of the impact of vaccination on the age-serological profiles for rubella antibodies over the period 1984-2030 in the United Kingdom in the male $(c)$ and female $(b)$ segments of the population. The basis of the predictions is described in ref. 67 . In the period $1970-84$ a single-stage UK policy was in operation (vaccination of teenage girls between the ages of 10-15 yr, with the observed vaccination rates for England and Wales). In 1985 a two-stage (UK-US) policy was begun (vaccination of teenage girls at 1984 rates, vaccination of $60 \%$ of 2 -yr-old boys and girls) to be maintained up to 2030. Note how the introduction of the two-stage policy alters the level of herd immunity in both the male and female segments of the population. The age axis records the intervals $0,0.5,1.5,2.5, \ldots 49.5 \mathrm{yr}$. 
hence the creation of herd immunity may require repeated cohort immunization. Cost factors and other practical issues are therefore likely to be of great importance in developing countries when considering the benefits of mass vaccination versus mass chemotherapy.

\section{Conclusions}

Many difficulties surround the attainment of sufficient levels of herd immunity to eradicate common infections in developed and developing countries. Theory can define the level of vaccination coverage required for elimination, but success in practice depends on economic and motivational issues. Vaccines against protozoan and helminth parasites will undoubtedly be of importance in the future (certainly to the Western traveller to tropical regions, but less certainly to those who live in these areas), and research towards their development must remain a priority. This emphasis, however, should not distract attention from the importance of epidemiological research. Effective communityGeneva, 1980)

2. Katz, S. L. Pediatrics 71, 653-654 (1983).

3. Centres for Disease Control. Morbidity Mortality Weekly Rep. 32, 1-125 (1983).

4. Noah, N. D. Br. med. J. 289, 1476 (1984).

5. Brook, C. G. D. Br. med. J. 286, 1082-1083 (1983)

6. Editorial Lancet it, 1343-1344 (1983).

7. Campbell, A. G. M. Archs Dis. Childh. 58, 3-5 (1983)

8. Hinman, A. R. Rev. infect. Dis. 4, 933-936 (1982)

9. Walsh, J. A. \& Warten, K. S. New Engl. J. Med. 301, $967-974$ (1979)

0. Creese, A. L. \& Henderson, R. H. Bull. Wld Hith Org. 58, 491-497 (1980).

11. Henderson, R. H. Wid Hith February/March 32-35 (1979).

12. De Quadros, C. A. Proc. R Soc. B208, 111-118 (1980).

13. Jilg, W. et al. Lancet ii, 1174-1175 (1984).

14. Walgate, R. Nature 312,190 (1984)

15. Weatherall, D. Lancet if, 1440-1443 (1984).

6. Mitchison, N. A. Nature 308, $112-113$ (1984)

17. Bull. Wid. Hith Org. 61, $81-92$ (1983)

18. Maddox, J. Nature 310, 541 (1984).

19. Brown, K. Nature 311, 300 (1984).

20. Editorial Lancet i, 1053-1055 (1984).

1. Poporic, M., Sorngadharan, M. G., Read, E. \& Gallo, R. C. Science 224, 497-500 (1984).

22. Walsh, J. A. Rev. infect. Dis. 5, 330-340 (1983)

23. Anderson, R. M. \& May, R. M. Science 215, 1053-1060 (1982)

24. Anderson. R. M. \& May, R. M. J. Hyg. Camb. 90, 259-325 (1983).

25. Anderson, R. M. \& May, R. M. J. Hyg., Camb. 94, 365-436 (1985)

26. Anderson, R. M. \& May, R. M. IMA J. math. Appl. Biol Med. 1, 233-266 (1984).

27. Dietz, K. Lect. Notes Biomath. 11, 1-15 (1976).

28. Hethcote, H. W. Am. J. Epidemiol. 117, 2-13 (1983)

29. Hoppensteadt,. H. C. J. Franklin Inst. 297, 325-333 (1974).

30. Schenzle, D. J. IMA J. math. Appl Biol. Med. 1, 169-191 (1984)

31. Anderson, R. M. \& May, R. M. Nature 280, 361-367 (1979)

32. May, R. M. \& Anderson, R. M. Nature 280, 455-461 (1979).

33. Anderson, R. M. in Population Biology of Infectious Diseases (eds Anderson, R. M. \& May, R. M.) 149-176 (Springer, Berlin, 1982).

34. Anderson, R. M. (ed.) Population Dynamics of Infectious Diseases (Chapman \& Hall, London, 1982).

35. Dietz, K. in Epidemiology (eds Ludwig, D. \& Cooke, . K.L. 104-121 (Society for Industrial and Applied Mathematics, Philadelphia, 1975).

36. Ross, R. Br. med. J. 1, 546-547 (1915)

37. Macdonald, G. The Epidemiology and Control of Malaria (London, Univesity Press, 1957).

38. Crjetanovic, B., Grab, B. \& Uemura, K. Bull Wid Hlth Org. 56, 1-143 (1978).

39. Anderson, R. M. \& May, R. M. Nature 297, 557-563 (1982).

40. Anderson, R. M. \& May, R. M. Adv. Parasit. 25, 1-101 (1985)

41. Bartlett, M. S. Proc. 3rd Berkeley Sump. Math statist. Probabilities 4, 81-109 (1956).

42. Cliff, A. D., Haggett, P., Ord, J. K. \& Versey, G. R. Spatial Diffusion: A Historical Geograph of Epidemics in an Island Community (Cambridge University Press, 1981).

43. Bartlett, M. S. J. R. statist. Soc. A120, 48-70 (1957)

44. Bartiet, M. S. Stochastic Population Models in Ecology and Epidemiology (Methuen, London, 1960).

4S. Hethcote, H., Stech, H. W. \& Van den Driessche, P. SIAM J. appl. Math. 40, 1-9 (1981)

46. Schwartz, I. B. \& Smith, H. L. J. Math. Biol. 18, 233-254 (1983).

47. London, W. P. \& Yorke, J. A. Am. J. Epidemiol 98, 453-468 (1973).

48. Yorke, J. A. \& London, W. P. Am. J. Epidemiol 98, 469-482 (1973)

49. Yorke, J. A., Nathanson, N., Pianigiani, G. \& Martin, J. Am. J. Epidemiol 109, 103-123 (1979).

50. Aron, J. L. \& Schwartz, I. B. J theor. Biol 110, 665-679 (1984).

51. Anderson, R. M., Grenfell, B. T. \& May, R. M. J. Hyg., Camb. 93, 587-608 (1984).

52. May, R. M. \& Anderson, R. M. Math. Biosci. (in the press)

53. Smith, C. E. Proc. $R$. Soc. Med. 63, $1181-1190$ (1970).

54. Albrecht, P., Ennis, F. A., Saltzinon, E. J. \& Krugman, S. J. Pediat. 91, 715-718 (1978).

55. Heyman, D. L., Mayben, G. K., Murphy, K. R., Guyer, B. \& Foster, S. O. Lancet if, 1470-1471 (1983).

56. Expanded Programme for Immunization: Programme Statement (WHO, Geneva, 1976)

57. Editorial Lancet i, 438-439 (1985).

58. Risi, J. B. Assign. Child 65/68, 137-157 (1984)

59. Warren, K. S. \& Mahmoud, A. A. F. Tropical and Geographical Medicine (McGraw-Hill, New York, 1984).

60. Breman, J. G. \& Arita, I. New Engl. J. Med. 303, 1263-1273 (1980).

61. Anderson, R. M. \& May, R. M. New Scient. 96, 410-415 (1982).

62. Fine, P. E. M. \& Clarkson, J. E. Int. J. Epidemiol. 11, 15-25 (1982)

63. Fine, P. E. M. \& Clarkson, J. E. J. Hyg., Camb. 92, 21-36 (1984).

64. Gregg, N. M. Trans. opthal Soc. Aust. 3, 35-36 (1941). wide control of many of the world's major infections is likely to depend on integrated approaches, involving some combination of selective or targeted vaccination and chemotherapy (directed towards those most predisposed to infection and morbidity), vector control and efforts to improve nutrition, hygiene, sanitation and education. Insights founded on an understanding of the population dynamics of infection will, therefore, be of great importance in any quantitative assessment of control policy. The recent convergence of mathematical theory and observation in epidemiology has created a powerful set of tools for the design and evaluation of community-based programmes of disease control, provided they are used sensibly. At present the potential value of these techniques is not widely appreciated.

We thank the Rockefeller Foundation (R.M.A. and R.M.M.), the Department of Health and Social Security, UK (R.M.A.), and the NSF (grant BSR83-03772 to R.M.M.) for financial support.

65. Hansow, J. B. Dudgeon, J. A. Viral Diseases of the Foetus and Newborn (Saunders, London, 1978).

66. Brunell, P. A. in Pediatric Infectious Diseases (eds Feigin, R. D. \& Cherry, J. D. ) 123-1235 (Saunders, London, 1981).

67. Anderson, R. M. \& Grenfell, B. T. J. Hyg., Camb. (in the press).

68. Sydenstricker, E. \& Hedrick, A. W. US publ Hith Rep. 44, 1537-1543 (1928)

69. Fine, P. E. M. \& Clarkson, J. A. Int. J. Epidemiol. 11, 5-14 (1982)

70. Black, F. J. Dis. Childh. 103, 242-249 (1962)

71. Kermack, W. D. \& McKendrick, A. G. Proc. R. Soc. Al15, 13-23 (1927).

72. Hethcote, H. W. Theor. Popul Biol 14, 338-349 (1978).

73. Becker, N. Biomerrics 35, 295-305 (1979).

74. May, R. M. \& Anderson, R. M. Math. Biosci. 72, 83-111 (1984).

75. Schenzle, D. Lect. Notes Biomath. 57, 171-178 (1985).

76. Lajmanovich, A. \& Yorke, J. A. Math. Biosci 28, 221-236 (1976)

77. Post, N. M., DeAngelis, D. L. \& Travis, C. C. Math. Biosci. 63, 289-302 (1983).

78. Bodmer, W. J. R. Coll. Phys., Lond. 14, 43-50 (1980)

79. Brady, J. A., Sever, J. L., McAlister, R., Schiff, G. M. \& Cutting, R. J. Am. med. Ass. 191, 619-626 (1965).

80. Christensen, P. E. et al. Danish med. Bull 1, 2-6 (1953).

81. Macgregor, J. D., Macdonald, J., Ingram, E. A., McDonnell, M. Marshall, B. Br. med. J. 282, 434-436 (1981).

82. Pandy, J. P., Zollinger, W. D., Fudenberg, H. H. \& Loadholt, C. B. Clin. Invest. 68, 1378-1380 (1981).

83. Spencer, M. J. et al. Immunogenerics 4, 365-372 (1977).

84. Kato, S., Muranaka, S., Takakura, I., Kimara, M. \& Tsum, K. Antigen 19, 140-145 (1982).

85. Hosking, C. S. \& Robertson, D. M. Clinics Immun. Allergy 1, 631-639 (1981).

86. Barbour, A. D. Trans. R. Soc. trop. Med. Hyg. 72, 6-15 (1978)

87. Dietz, K. Lect. Notes Biomath. 39, 264-277 (1980)

88. Hethcote, H. W. \& Yorke, J. A. Lect. Notes Biomath. 56, 1-105 (1984).

89. Conrad, G. L., Klevis, G. S., Rush, 8. \& Darrow, W. W. Sex. Transmitted Dis. 8, 241-244 (1981).

90. Rothenburg, R. B. Am. J. Epidemiol. 117, 688-694 (1983)

91. Bloom, A. L. Lancet i, 1452-1455 (1984).

92. Safai, B. et al. Lancet $\mathrm{i}, 1438-1440$ (1984).

93. Goedert, J. J. et al. Lancet it, 711-715 (1984).

94. Fine, P. E. M. \& Clarkson, J. A. Int. J. Epidemiol. 11, 5-14 (1982).

95. Morley, D. Proc. R. Soc. Med. 57, 846-849 (1969).

96. Black, F. L. in Viral diseases of South East Asia and Westem Pacific (ed. Mackenzie, J.) 297-307 (Academic, Sydney, 1982)

97. Heyman, D. L. et al. Br. med. J. 285, $531-533$ (1982).

98. Kok, P. W., Kenya, P. R. \& Ensering, H. Trans. R Soc. trop. Med. Hyg. 77, 171-176 (1983).

99. McLean, A. Lect. Notes Biomath. (in the press).

100. Newark, P. Nature 311, 510 (1984).

101. McAleer, W. J. et al. Nature 307, 178-180 (1984).

102. Francis, D. P. Rev. infect. Dis. 5, 321-329 (1983).

103. Stevens, C. E., Beasiey, R. P., Tsui, J. \& Lee, W. C. New Engl. J. Med. 292, 771-774 (1975).

104. Maupas, P. et al. Lancet 1, 289-292 (1981)

105. Coppel, R. L. et al. Nature 310, 789-792 (1984)

106. Phillip, M., Parkhouse, R. M. E. \& Ogilvie, B. M. Nature 287, 538-540 (1980).

107. Cross, G. A.M. Proc. R. Soc. B202, 55-68 (1978)

108. Turner, M. J. Parasitology 88, 613-621 (1984).

109. Deloron, P., Jaureguiberry, G., LeBrars, J. \& Coulanges, D. Lancet i, 444-446 (1985).

110. Warren, K. S. J. infect. Dis. 127, 595-561 (1978).

111. Parkhouse R M. E (ed) Parasitology $83(1984)$

112. Schad, G. A. \& Anderson, R. M. Science 228, 1537-1540 (1985)

113. Anderson, R. M. \& May, R. M. Nature 315, 439-496 (1985)

114. Grenfell, B. T. \& Anderson, R. M. J. Hyg., Camb. (in the press).

116. Morbidity and Mortality Weekly Report No. 30, 362-363 (Centers for Disease Control, Washington, 1981).

120. Clarke, M., Schild, G. C., Boursted, J., McGregor, I. A. \& Williams, K. Bull. Wh Hith Org. 58, $931-935$ (1980)

117. Black, F. L. J. Immun. 83, 74-83 (1959).

118. Shah, U., Banerji, X. L., Nanavati, A. N. D. \& Mehta, N. A. Bull. Wild Hith Org. 46, 130-138 (1972).

115. Molineaux, L. \& Gramiccia, G. The Garki Project (WHO, Geneva, 1980).

119. Maupas, P. \& Melnick, J. L. Prog. med. Virol. 27, 1-5 (1981).

120. Clarke, M., Schild, G. C., Boursted, J., McGregor, I. A. \& Williams, K. Bull. Whd Hith Org. 58, 931-935 (1980)

121. Lundstrom, R., Svedmyr, A., Hagbard, L. \& Kaijser, K. Acta paediat. scand. 56, 279-285 (1967).

122. Mortimer, P. P. Brit. med. J. 2, 1523-1524 (1978).

123. Anderson, M. J. J. Hyg., Camb. 89, 1-8 (1982). 